\title{
Crystal lattice vibrations and their coupling with magnetic correlations in $(\mathrm{Cu}, \mathrm{Co}) \mathrm{Sb}_{2} \mathrm{O}_{6}$
}

\author{
Damaris T. Maimone (PG), Eduardo Granado (PQ).
}

\begin{abstract}
In this work, the magnetic low dimensional systems $(\mathrm{Cu}, \mathrm{Co}) \mathrm{Sb}_{2} \mathrm{O}_{6}$ were studied. One of the motivations for this study is the understanding of the anomalous behavior in the reported magnetic susceptibility measurements. We studied these compounds through a spectroscopic technique capable of providing us information about the magnetic correlations and exchange interactions in first neighbors, namely Raman spectroscopy. In particular, we investigated the spin-phonon coupling for the Raman active modes of $\mathrm{CuSb}_{2} \mathrm{O}_{6}$ and performed preliminary measurements in $\mathrm{CoSb}_{2} \mathrm{O}_{6}$.
\end{abstract}

Key words: Low-dimension magnetism, Raman Spectroscopy, spin-phonon coupling.

\section{Introduction}

Low dimensional systems have been thoroughly studied both experimentaly and theoreticaly, due to their peculiar magnetic properties. In this work, we studied the magnetic low dimensional systems $\mathrm{CoSb}_{2} \mathrm{O}_{6}$ and $\mathrm{CuSb}_{2} \mathrm{O}_{6}$. Even though these compounds present a similar crystal structure, their magnetic susceptibility (MS) show different magnetic behaviors. It is possible to notice that the substitution of Co per Cu drives the change of magnetic structure from antiferromagnetic(AFM) bidimensional[2] to AFM one dimensional[1]. This anomalus behavior in the MS is still an open question. In this work we investigated these compounds through a spectroscopic technique capable of providing us the information about spin correlations and exchange interactions in first neighbors. The technique used here was Raman spectroscopic, that is sensitive to the lattice vibrations and their coupling with magnetic correlations. The spin-phonon coupling manifests as a change in the vibration frequencies due to a modulation in the magnetic energy. If one follows the phonon energies as a function of temperature it is possible to obtain information about the spin correlations.

For the Cu-based sample, it was reported a structural transition at $\mathrm{T}=380 \mathrm{~K}[1]$, where it goes from monoclinic (P21/n) to tetragonal (P42/mnm). Using Raman spectroscopy we could also see the transition, signed as a division of the peak at 648 $\mathrm{cm}-1$ ( $\mathrm{T}=400 \mathrm{~K})$ into two peaks $635 \mathrm{~cm}-1$ and 678 $\mathrm{cm}-1$ ( $\mathrm{T}=296 \mathrm{~K})$, as shown in Fig. 1 .

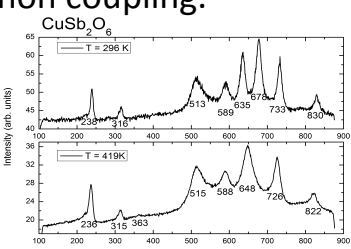

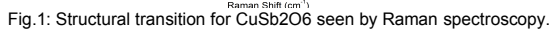

In order to follow the frequency as a function of temperature and then investigate the spin correlations, we took several spectra at different temperatures. The result for two different peaks are shown in Fig.2. It is possible to see an anomaly near $\mathrm{T}=100 \mathrm{~K}$, which we ascribe to the spin-phonon coupling.

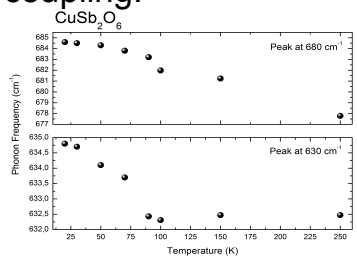

Fig.2: Following the frequency as a function of temperature to see the spin correlations and then the anomaly for CuSb2O6.

\section{Conclusions}

Raman scattering measurements were performed on $\mathrm{CuSb}_{2} \mathrm{O}_{6}$. The structural transition at $380 \mathrm{~K}$ manifests as a splitting of the phonon peak at 648 $\mathrm{cm}-1$ into two peaks. Anomalies in some phonon frequencies were observed below $\sim 100 \mathrm{~K}$, which we ascribed to a spin-phonon coupling. These results indicate interesting physics for these compounds, which are being investigated in more detail during my Masters dissertation work.

\section{Acknowledgement}

I would like to thank my professor for all the support he gave and is giving me. I also would like to thank CNPq/PIBIC for the scholarship.

\section{References}

[1] A. Nakua, H. Yun, J. N. Reimers," J. E. Greedan, C. V. Stagers, Journal of Solid State Chemistry 91, 105(1991). [2] J. N. Reimers, J. E. Greedan, C. V. Stager, AND R. Kremer, Journal of Solid State Chemistry 83, 20(1989). 